\title{
Suppression of current in transport through parallel double quantum dots
}

\author{
Tae-Suk Kim ${ }^{a, b}$ and S. Hershfield ${ }^{b}$ \\ a Institute of Physics and Applied Physics, Yonsei University, Seoul 120-749, Korea \\ ${ }^{b}$ Department of Physics, University of Florida, Gainesville FL 32611-8440
}

(October 28, 2018)

\begin{abstract}
We report our study of the I-V curves in the transport through the quantum dot when an additional quantum dot lying in the Kondo regime is side-connected to it. Due to the Kondo scattering off the effective spin on a side-connected quantum dot the conductance is suppressed at low temperatures and at low source-drain bias voltages. This zero-bias anomaly is understood as enhanced Kondo scattering with decreasing temperature.
\end{abstract}

72.10.Fk, 72.15.Qm

\section{INTRODUCTION}

Advances in nanotechnology have made it possible to fabricate quantum dots or artificial atoms and to study their transport properties. Due to the confinement and the Coulomb interaction in the quantum dot, charge and spin can be quantized. Quantization of electron charge leads to the almost periodic variation of conductance as a function of the number of electrons $N$ or the gate voltage. A conductance peak is observed whenever the charge fluctuation is allowed or the $N$ - and $(N+1)$-states become energetically degenerate. Otherwise electrons in the electrodes cannot hop into a quantum dot due to the strong Coulomb repulsion. This Coulomb blockade leads to vanishing conductance between two neighboring conductance peaks. Novel Kondo effects can enhance the conductance in the Coulomb blockade region when the number of electrons at the quantum dot is odd and at least one electron spin is unpaired. The unpaired $S=1 / 2$ in the quantum dot cap be screened by the electrons in the external electrodes connected to the quantum dot. This theoretical predictionl of the novel nonequilibrium Kondo effects in quantum dot systems was confirmed experimentally recently 2 t. In the conventional bulk Kondo systems where magnetic ions are doped into normal metals the study of the Kondo effects was confined to the equilibrium state. In quantum dot systems the nonequilibrium situation is easily controlled and model parameters can be varied by adjusting the gate voltages.

The study of the Kondo effect in the quantum dot systems has been diversified to see the different aspects. The quantum dots are inserted in the Aharonov-Bohm ring to study the effect of many-body interaction on the persistent current 5 . When electrons are scattered off the magnetic impurity, they experience a phase shift $\pi / 2$ at the Fermi energy due to the Kondo effect. The direct measurement of this phase shift is not possible in the bulk systems. Using a quantum dot inserted in the Aharonov-Bohm ring the phase shift due to the Kondo effect was studied theoretically 6 and measured experimentallyt. Serial double quantum dots were also studied in the Kondo regimel. Enhanced conductance was observed $\mathrm{B}$ in an otherwise Coulomb blockade region at the spin singlet-to-triplet transition point when the number of electrons on a quantum dot is even. The ground state can be either spin-singlet or spin-triplet depending on the geometry of the confinement potential. Perpendicular magnetic fields can induce a transition of the ground state between spin-singlet and spin-triplet. This-novel Kondo effects have been interpreted as coming from the enhanced Kondo temperature at the transition point 10 .

In this paper we study theoretically the transport properties of parallel double quantum dots as depicted in Fig.1. One active quantun dot is connected to the source and drain electrodes and the other quantum dot is side-connected to the active quantum dot. Since the energy levels in two quantum dots can be controlled separately using the gate voltage, different transport regimes can be probed. In this work we present our study of the I-V characteristics of this system when the active quantum dot lies in the conductance peak region while the side-connected quantum dot in the Coulomb blockade region. In this case charge fluctuations are suppressed and an effective spin $S=1 / 2$ arises in a side-connected dot. Electrons passing through the active dot experience the Kondo scattering off an effective spin in a side-connected dot. We find that the linear response conductance is suppressed at low temperature due to the enhanced Kondo scattering off spin $S=1 / 2$. The spectral function at a side-connected dot develops a Kondo resonance peak as the temperature is lowered. On the other hand the spectral function at the active dot becomes depleted near $\omega=0$ with decreasing temperature. We obtained these results using the nonequilibrium Green's function method combining with the non-crossing approximation (NCA).

This paper is organized as follows. In section II we introduce our model Hamiltonian and present the formulation of current passing through an active dot. The relevant Green's functions in nonequilibrium are formulated in section III using the non-crossing approximation (NCA) which is modified for our system. Numerical results, solving the NCA 
equations self-consistently, are presented in section IV and conclusion is included in section V.

\section{PARALLEL DOUBLE QUANTUM DOTS}

\section{A. Model Hamiltonian}

Consider the parallel double quantum dots shown in Fig. 1. In this experimental geometry, the transfer of electrons from one electrode to the other is realized by hopping on and off an active quantum dot (the site $A$ ). The other dot (the site $S$ ) is side-connected to the active dot by hopping. Assuming that the energy level spacing is large enough compared to the level-broadening due to the tunneling into two electrodes, the model Hamiltonian can be written as

$$
\begin{aligned}
H & =H_{\mathrm{el}}+H_{\mathrm{qdot}}+H_{1}, \\
H_{\mathrm{el}} & =\sum_{p=L, R} \sum_{\vec{k} \alpha} \epsilon_{p \vec{k}} c_{p \vec{k} \alpha}^{\dagger} c_{p \vec{k} \alpha}, \\
H_{\mathrm{qdot}} & =\sum_{i=A, S} \sum_{\alpha} E_{i} d_{i \alpha}^{\dagger} d_{i \alpha}+\sum_{i=A, S} U_{i} n_{i \uparrow} n_{i \downarrow}, \\
H_{1} & =\sum_{p=L, R} \sum_{\vec{k} \alpha}\left[V_{p}(\vec{k}) c_{p \vec{k} \alpha}^{\dagger} d_{A \alpha}+V_{p}^{*}(\vec{k}) d_{A \alpha}^{\dagger} c_{p \vec{k} \alpha}\right]+\sum_{\alpha}\left[W d_{A \alpha}^{\dagger} d_{S \alpha}+W^{*} d_{S \alpha}^{\dagger} d_{A \alpha}\right] .
\end{aligned}
$$

The electron creation operator of spin $\alpha$ in the electrode $p=L, R$ is $c_{p \vec{k} \alpha}^{\dagger}$. The index $p=L(R) \operatorname{denotes}$ the left(right) external electrode. Both electrodes are assumed to be described by the Lorentzian density of states (DOS) with the energy dispersion $\epsilon_{p \vec{k}} \equiv \epsilon_{\vec{k}}+\mu_{p} \cdot \mu_{L, R}= \pm \frac{1}{2} \mathrm{eV}$ is the chemical potential shift due to the bias voltage applied to the electrodes. $d_{i \alpha}^{\dagger}$ is the electron creation operator in a quantum dot $i=A, S$ with $A(S)$ labeling a active (side-connected) dot, respectively. $U_{i}$ is the on-site electron-electron Coulomb interaction in a dot $i$.

When $U_{A}=U_{S}=0$, two dots become resonant levels and the solution can be found analytically (see Appendix A). The model becomes nontrivial when one of on-site Coulomb interactions or both are nonzero. In this paper we are going to study the case of $U_{A}=0$ and $U_{S} \neq 0$ in detail. When $U_{S}$ is the largest of all the model parameters and $-E_{S} \gg|W|$, charge fluctuations in a side-connected dot can be neglected and the side-connected dot can be treated as an effective spin $S \overline{\bar{T}}^{1 / 2}$. In this case removing the charge degrees of freedom in a side-connected dot (Schrieffer-Wolf transformation 11) the interaction between two dots can be derived.

$$
H_{A S}=J_{A S} \vec{S}_{S} \cdot \sum_{\alpha \beta} d_{A \alpha}^{\dagger} \frac{1}{2} \vec{\sigma}_{\alpha \beta} d_{A \beta}, \quad J_{A S}=\frac{2|W|^{2}}{-E_{S}}+\frac{2|W|^{2}}{U_{S}+E_{S}}
$$

Here $\vec{S}_{S}=\sum_{\alpha \beta}\left|S ; \alpha>\frac{1}{2} \vec{\sigma}_{\alpha \beta}<S ; \beta\right|$ represents the spin degrees of freedom in a side-connected dot in the absence of charge fluctuations. Electrons, flowing from the left electrode to the right, at the active dot will experience the Kondo scattering off an effective spin of a side-connected dot. Appendix $\mathrm{B}$ contains the details of the $U_{A} \neq 0$ and $U_{S} \neq 0$ case.

\section{B. Formulation of Current}

The current operator can be defined as a variation of the number operators of electrons per unit time leaving one electrode $(p=L, R)$.

$$
\hat{I}_{p} \equiv-e\left(-\dot{N}_{p}\right)=\frac{e}{i \hbar}\left[N_{p}, H\right]=\frac{e}{i \hbar} \sum_{\vec{k} \alpha}\left[V_{p}(\vec{k}) c_{p \vec{k} \alpha}^{\dagger} d_{A \alpha}-H . c .\right] .
$$

The measured current is given by the thermal average of the above current operator and can be written in terms of the lesser Green's functions out of equilibrium 12 .

$$
I_{p}(t)=-i e \sum_{\vec{k} \alpha}\left[V_{p}(\vec{k}) G_{A c p}^{<}(\vec{k} ; t, t)-V_{p}^{*}(\vec{k}) G_{c p A}^{<}(\vec{k} ; t, t)\right] .
$$


The current can be expressed in terms of Green's functions for the conduction electrons and quantum dots using the Dyson equations for the mixed Green's functions

$$
\begin{aligned}
G_{c p A}\left(\vec{k} ; t, t^{\prime}\right) & \equiv \frac{1}{i \hbar}\left\langle T c_{p \vec{k} \alpha}(t) d_{A \alpha}^{\dagger}\left(t^{\prime}\right)\right\rangle \\
& =V_{p}(\vec{k}) \int_{C} d t_{1} G_{c p}\left(\vec{k} ; t, t_{1}\right) D_{A}\left(t_{1}, t^{\prime}\right), \\
G_{A c p}\left(\vec{k} ; t, t^{\prime}\right) & \equiv \frac{1}{i \hbar}\left\langle T d_{A \alpha}(t) c_{p \vec{k} \alpha}^{\dagger}\left(t^{\prime}\right)\right\rangle \\
& =V_{p}^{*}(\vec{k}) \int_{C} d t_{1} D_{A}\left(t, t_{1}\right) G_{c p}\left(\vec{k} ; t_{1}, t^{\prime}\right), \\
i \hbar D_{i}\left(t, t^{\prime}\right) & =<T d_{i \alpha}(t) d_{i \alpha}^{\dagger}\left(t^{\prime}\right)>, \quad i=A, S .
\end{aligned}
$$

Using analytic continuation to the real-time axis 13 , we can find the lesser part of $G_{A c p}\left(\vec{k} ; t, t^{\prime}\right)$

$$
G_{A c p}^{<}\left(\vec{k} ; t, t^{\prime}\right)=V_{p}^{*}(\vec{k}) \int d t_{1}\left[D_{A}^{<}\left(t, t_{1}\right) G_{c p}^{a}\left(\vec{k} ; t_{1}, t^{\prime}\right)+D_{A}^{r}\left(t, t_{1}\right) G_{c p}^{<}\left(\vec{k} ; t_{1}, t^{\prime}\right)\right] .
$$

The current can be expressed as

$$
\begin{aligned}
I_{p} & =-i e \frac{1}{V} \sum_{\vec{k} \alpha}\left|V_{p}(\vec{k})\right|^{2} \int \frac{d \omega}{2 \pi}\left\{D_{A}^{<}(\omega)\left[\Sigma_{c p}^{a}(\omega)-\Sigma_{c p}^{r}(\omega)\right]+\Sigma_{c p}^{<}(\omega)\left[D_{A}^{r}(\omega)-D_{A}^{a}(\omega)\right]\right\} \\
& =-i e \sum_{\alpha} \int \frac{d \omega}{2 \pi}\left|D_{A}^{r}(\omega)\right|^{2}\left\{\Sigma_{A}^{<}(\omega)\left[\Sigma_{c p}^{a}(\omega)-\Sigma_{c p}^{r}(\omega)\right]+\Sigma_{c p}^{<}(\omega)\left[\Sigma_{A}^{r}(\omega)-\Sigma_{A}^{a}(\omega)\right]\right\} .
\end{aligned}
$$

Here $\Sigma_{A}$ is the self-energy for the active $\operatorname{dot} A$ and the following equations are used

$$
\begin{aligned}
\Sigma_{c p}\left(t, t^{\prime}\right) & =\frac{1}{V} \sum_{\vec{k}}\left|V_{p}(\vec{k})\right|^{2} G_{c p}\left(\vec{k} ; t, t^{\prime}\right), \\
\Sigma_{c p}^{r, a}(\omega) & =\int \frac{d \epsilon}{\pi} \frac{\Gamma_{p}(\epsilon)}{\hbar \omega-\epsilon \pm i \delta}, \\
\Sigma_{c p}^{<,>}(\omega) & =2 \Gamma_{p}(\omega) f_{p}( \pm \omega), \\
\Gamma_{p}(\omega) & \equiv \frac{\pi}{V} \sum_{\vec{k}}\left|V_{p}(\vec{k})\right|^{2} \delta\left(\hbar \omega-\epsilon_{p \vec{k}}\right) .
\end{aligned}
$$

The above equation for the current is quite general and can be used as a starting point in reducing the expression of current in specific cases.

The average number of electrons at a side-connected $\operatorname{dot} S$ should remain the same in a steady state. This condition is essential to get the right expression for the current flowing from the left to the right electrodes. That is, the net current flowing out of the dot $S$ should vanish for

$$
I_{S}=\frac{e}{i \hbar} \sum_{\alpha}\left[W^{*}<d_{S \alpha}^{\dagger} d_{A \alpha}>-W<d_{A \alpha}^{\dagger} d_{S \alpha}>\right]=-i e \sum_{\alpha}\left[W^{*} G_{A S}^{<}(t, t)-W G_{S A}^{<}(t, t)\right] .
$$

The current $I_{S}$ was expressed in terms of the mixed Green's functions

$$
\begin{aligned}
& i \hbar G_{A S}\left(t, t^{\prime}\right)=<T d_{A \alpha}(t) d_{S \alpha}^{\dagger}\left(t^{\prime}\right)>, \\
& i \hbar G_{S A}\left(t, t^{\prime}\right)=<T d_{S \alpha}(t) d_{A \alpha}^{\dagger}\left(t^{\prime}\right)>.
\end{aligned}
$$

These mixed Green's functions be written as

$$
\begin{aligned}
& G_{A S}\left(t, t^{\prime}\right)=W \int_{C} d t_{1} \tilde{D}_{A}\left(t, t_{1}\right) D_{S}\left(t_{1}, t^{\prime}\right)=W \int_{C} d t_{1} D_{A}\left(t, t_{1}\right) \tilde{D}_{S}\left(t_{1}, t^{\prime}\right), \\
& G_{S A}\left(t, t^{\prime}\right)=W^{*} \int_{C} d t_{1} \tilde{D}_{S}\left(t, t_{1}\right) D_{A}\left(t_{1}, t^{\prime}\right)=W^{*} \int_{C} d t_{1} D_{S}\left(t, t_{1}\right) \tilde{D}_{A}\left(t_{1}, t^{\prime}\right) .
\end{aligned}
$$


Here $D_{A}$ and $D_{S}$ are the fully dressed Green's functions of the dots $A$ and $S$, respectively.

$$
\begin{aligned}
& i \hbar D_{A}\left(t, t^{\prime}\right)=<T d_{A \alpha}(t) d_{A \alpha}^{\dagger}\left(t^{\prime}\right)>, \\
& i \hbar D_{S}\left(t, t^{\prime}\right)=<T d_{S \alpha}(t) d_{S \alpha}^{\dagger}\left(t^{\prime}\right)>.
\end{aligned}
$$

On the other hand $\tilde{D}_{A}$ and $\tilde{D}_{S}$ are the corresponding Green's functions which cannot be separated into two parts by removing one tunneling matrix $W$. Using analytic continuation the current $I_{S}$ can be written as

$$
\begin{aligned}
I_{S} & =-i e|W|^{2} \sum_{\alpha} \int \frac{d \omega}{2 \pi}\left\{\left[\tilde{D}_{A}^{r}(\omega)-\tilde{D}_{A}^{a}(\omega)\right] D_{S}^{<}(\omega)-\left[D_{S}^{r}(\omega)-D_{S}^{a}(\omega)\right] \tilde{D}_{A}^{<}(\omega)\right\} \\
& =e \sum_{\alpha} \int \frac{d \omega}{2 \pi} 2|W|^{2}\left|\tilde{D}_{A}^{r}(\omega)\right|^{2}\left[\pi \tilde{\Sigma}_{A}^{<}(\omega) A_{S}(\omega)+\operatorname{Im}\left\{\tilde{\Sigma}_{A}^{r}(\omega)\right\} D_{S}^{<}(\omega)\right] .
\end{aligned}
$$

Since $I_{S}=0$ in steady state, we find the equation relating the lesser part to the retarded part of the Green's function or the spectral function $A_{S}$ for the $\operatorname{dot} S$.

$$
\begin{aligned}
D_{S}^{<}(\omega) & =\pi \frac{\tilde{\Sigma}_{A}^{<}(\omega)}{-\operatorname{Im} \tilde{\Sigma}_{A}^{r}(\omega)} A_{S}(\omega), \\
f_{\text {eff }}(\omega) & =\frac{\tilde{\Sigma}_{A}^{<}(\omega)}{-2 \operatorname{Im} \tilde{\Sigma}_{A}^{r}(\omega)} .
\end{aligned}
$$

That is, the condition that no net current flows out of the $\operatorname{dot} S$ determines its nonequilibrium thermal distribution function. The current $I_{S}$ can be expressed in another form

$$
\begin{aligned}
I_{S} & =-i e|W|^{2} \sum_{\alpha} \int \frac{d \omega}{2 \pi}\left\{\left[D_{A}^{r}(\omega)-D_{A}^{a}(\omega)\right] \tilde{D}_{S}^{<}(\omega)-\left[\tilde{D}_{S}^{r}(\omega)-\tilde{D}_{S}^{a}(\omega)\right] D_{A}^{<}(\omega)\right\} \\
& =e \sum_{\alpha} \int \frac{d \omega}{2 \pi} 2|W|^{2}\left|D_{A}^{r}(\omega)\right|^{2}\left[\pi \Sigma_{A}^{<}(\omega) \tilde{A}_{S}(\omega)+\operatorname{Im}\left\{\Sigma_{A}^{r}(\omega)\right\} \tilde{D}_{S}^{<}(\omega)\right]
\end{aligned}
$$

\section{Interacting Case: $U_{A}=0$ and $U_{S} \neq 0$}

When $U_{A}=0$ and $U_{S} \neq 0$, the active $\operatorname{dot} A$ becomes a resonant level and the side-connected dot $S$ is strongly correlated. This model Hamiltonian may describe the situation that the $\operatorname{dot} A$ lies in the conductance peak region and the $\operatorname{dot} S$ in the Kondo or Coulomb blockade region. Green's functions of the two dots are given by the Dyson equations with the self-energies being given by

$$
\begin{aligned}
& \Sigma_{A}\left(t, t^{\prime}\right)=\Sigma_{c}\left(t, t^{\prime}\right)+W^{2} \tilde{D}_{S}\left(t, t^{\prime}\right), \\
& \Sigma_{S}\left(t, t^{\prime}\right)=\Sigma_{U}\left(t, t^{\prime}\right)+W^{2} \tilde{D}_{A}\left(t, t^{\prime}\right) .
\end{aligned}
$$

Here $\Sigma_{c}\left(t, t^{\prime}\right)=\Sigma_{c L}\left(t, t^{\prime}\right)+\Sigma_{c R}\left(t, t^{\prime}\right)$ [equation (2.8a)] is the self-energy coming from tunneling into left and right electrodes, $\Sigma_{U}\left(t, t^{\prime}\right)$ is the self-energy for a dot $S$ due to the on-site Coulomb interaction, and the auxillary Green's functions are defined by the Dyson equations

$$
\begin{aligned}
& \tilde{D}_{A}\left(t, t^{\prime}\right)=D_{A 0}\left(t, t^{\prime}\right)+\int_{C} d t_{1} \int_{C} d t_{2} \tilde{D}_{A}\left(t, t_{1}\right) \Sigma_{c}\left(t_{1}, t_{2}\right) D_{A 0}\left(t_{2}, t^{\prime}\right), \\
& \tilde{D}_{S}\left(t, t^{\prime}\right)=D_{S 0}\left(t, t^{\prime}\right)+\int_{C} d t_{1} \int_{C} d t_{2} \tilde{D}_{S}\left(t, t_{1}\right) \Sigma_{U}\left(t_{1}, t_{2}\right) D_{S 0}\left(t_{2}, t^{\prime}\right) .
\end{aligned}
$$

From the self-energy equations we find

$$
\begin{aligned}
\Sigma_{A}^{<}(\omega) & =2 \Gamma_{L}(\omega) f_{L}(\omega)+2 \Gamma_{R}(\omega) f_{R}(\omega)+W^{2} \tilde{D}_{S}^{<}(\omega), \\
\Sigma_{A}^{r}(\omega)-\Sigma_{A}^{a}(\omega) & =-2 i\left[\Gamma_{L}(\omega)+\Gamma_{R}(\omega)\right]+|W|^{2}\left[\tilde{D}_{S}^{r}(\omega)-\tilde{D}_{S}^{a}(\omega)\right] .
\end{aligned}
$$

Inserting all the equations into the expression of the current (2.7), we find 


$$
I_{p}=e \sum_{\alpha} \int \frac{d \omega}{2 \pi}\left\{4 \Gamma_{L}(\omega) \Gamma_{R}(\omega)\left|D^{r}(\omega)\right|^{2}\left[f_{\bar{p}}(\omega)-f_{p}(\omega)\right]+2|W|^{2} \Gamma_{p}(\omega)\left|D_{A}^{r}(\omega)\right|^{2}\left[\tilde{D}_{S}^{<}(\omega)-2 \pi f_{p}(\omega) \tilde{A}_{S}(\omega)\right]\right\}
$$

From the condition $I_{S}=0$, we have

$$
\begin{aligned}
\tilde{D}_{S}^{<}(\omega) & =\pi \frac{\Sigma_{A}^{<}(\omega)}{-\operatorname{Im} \Sigma_{A}^{r}(\omega)} \tilde{A}_{S}(\omega) \\
& =\frac{2 \pi\left[\Gamma_{L}(\omega) f_{L}(\omega)+\Gamma_{R}(\omega) f_{R}(\omega)\right]+\pi|W|^{2} \tilde{A}_{S}^{<}(\omega)}{\Gamma_{L}(\omega)+\Gamma_{R}(\omega)+\left.\pi W\right|^{2} \tilde{A}_{S}(\omega)} \times \tilde{A}_{S}(\omega) \\
& =2 \pi f_{\mathrm{eff}}(\omega) \tilde{A}_{S}(\omega) \\
f_{\mathrm{eff}}(\omega) & \equiv \frac{\Gamma_{L}(\omega) f_{L}(\omega)+\Gamma_{R}(\omega) f_{R}(\omega)}{\Gamma_{L}(\omega)+\Gamma_{R}(\omega)}
\end{aligned}
$$

It follows from the relation $I_{S}=0$ that $D_{S}^{<}(\omega)=2 \pi f_{\text {eff }}(\omega) A_{S}(\omega)$. The effective Fermi-Dirac function $f_{\text {eff }}(\omega)$ describes the nonequilibrium thermal distribution at the $\operatorname{dot} S$ and also at the active $\operatorname{dot} A$. [It can be shown from the equation (2.22).] The current flowing from left to right electrode becomes

$$
\begin{aligned}
I_{L} & =e \sum_{\alpha} \int \frac{d \omega}{2 \pi} T(\omega)\left[f_{R}\left(\omega-f_{L}(\omega)\right]\right. \\
T(\omega) & =4 \Gamma_{L}(\omega) \Gamma_{R}(\omega)\left|D_{A}^{r}(\omega)\right|^{2}\left[1+\frac{\pi|W|^{2} \tilde{A}_{S}(\omega)}{\Gamma_{L}(\omega)+\Gamma_{R}(\omega)}\right]=\frac{4 \pi \Gamma_{L}(\omega) \Gamma_{R}(\omega)}{\Gamma_{L}(\omega)+\Gamma_{R}(\omega)} A_{A}(\omega) .
\end{aligned}
$$

Note that the current $I_{L}$ consists of two contributions, coming from the direct path $(L \rightarrow A \rightarrow R)$ and the indirect path $(L \rightarrow A \rightarrow S \rightarrow A \rightarrow R)$, which are interfereing each other.

The current is written down in terms of the transmission coefficient, that is, in Landauer-Büttiker form. In turn the transmission coefficient is proportional to the spectral function of the active $\operatorname{dot} A$. To find the spectral function $A_{A}(\omega)$ we note that Green's functions of the two dots are related to each other by the equations

$$
D_{A}^{r}(\omega)=\tilde{D}_{A}^{r}(\omega)+|W|^{2} \tilde{D}_{A}^{r}(\omega) D_{S}^{r}(\omega) \tilde{D}_{A}^{r}(\omega) .
$$

To get the desired spectral function we have to calculate the Green's function $D_{S}^{r}$ at a side-connected dot and the auxillary Green's function $\tilde{D}_{A}^{r}$ at the active dot is given by the equation (2.17a). Due to the on-site Coulomb interaction at a side-connected $\operatorname{dot} S$, the calculation of $D_{S}^{r}(\omega)$ is highly non-trivial and requires a many-body calculation. In the next section we describe the non-crossing approximation in order to find the Green's function at a side-connected $\operatorname{dot} S$.

\section{NON-CROSSING APPROXIMATION (NCA)}

To study the I-V curves of our model system, we need to calculate the Green's function at a side-connected dot $S$ out of equilibrium. For this purpose we adopt the NGA which has been a very fruitful tool for the study of Anderson model in both equilibrium 14,15 and nonequilibrium 16,47 .

To begin with, we need to identify the effective continuum band for the dot $S$. In the Anderson model which describes the magnetic ions imbedded in normal metals, the self-energy can be written as a sum of two contributions.

$$
\Sigma_{f}(\omega)=\int \frac{d \epsilon}{\pi} \frac{\Gamma_{\mathrm{eff}}(\epsilon)}{\hbar \omega-\epsilon+i \delta}+\Sigma_{U}^{r}(\omega)
$$

The first term is the one-body contribution from tunneling into the conduction band and the second term $\Sigma_{U}$ is the many-body contribution from the on-site Coulomb interaction. On the other hand the self-energy of the dot $S$ in our model is given by

$$
\Sigma_{S}(\omega)=|W|^{2} \tilde{D}_{A}^{r}(\omega)+\Sigma_{U}^{r}(\omega) .
$$

Comparing the two self-energies (equations 3.1 and 3.2) we can identify that the effective Anderson hybridization function is given by the equation 


$$
\Gamma_{\text {eff }}(\epsilon)=-|W|^{2} \operatorname{Im} \tilde{D}_{A}^{r}(\epsilon)=-|W|^{2} \operatorname{Im}\left\{\frac{1}{\epsilon-E_{d}-\Sigma_{c}^{r}(\epsilon)}\right\}=\pi|W|^{2} \tilde{A}_{A}(\epsilon)
$$

The effective continuum band for the $\operatorname{dot} S$ is represented by the auxillary Green's function $\tilde{D}_{A}\left(t, t^{\prime}\right)$.

To find the Green's function for the dot $S$ we do a perturbative expansion in $W$. To begin with we represent the Fock space of the dot $S$ by the pseudo particle operators. In the large $U_{S}$ limit we may neglect the double occupation.

$$
d_{S \alpha} \rightarrow b^{\dagger} f_{\alpha}
$$

The boson operator $b$ annihilates the empty state and the pseudo fermion operator $f_{\alpha}$ destroys the singly occupied state at the $\operatorname{dot} S$. After replacing the electron operator $d_{S \alpha}$ by the pseudo particle operators, the occupation constraint has to be enforced: $Q=1$.

$$
Q=\sum_{\alpha} f_{\alpha}^{\dagger} f_{\alpha}+b^{\dagger} b
$$

This constraint is taken into account using the Lagrange multiplier method: $H \rightarrow H+\lambda(Q-1)$. The projection to the physical Hilbert space $Q=1$ leads to the following projection rule for the physical observables:

$$
\begin{aligned}
O(\omega) & =\frac{1}{Z_{S}} \lim _{\lambda \rightarrow \infty} e^{\beta \lambda} O_{\lambda}(\omega \rightarrow \omega+\lambda), \\
Z_{S} & =\lim _{\lambda \rightarrow \infty} e^{\beta \lambda}<Q>_{\lambda} .
\end{aligned}
$$

Here $O_{\lambda}(\omega)$ is a thermal average of observable operator $\hat{O}$ for the Hamiltonian $H+\lambda(Q-1)$. The Feynman diagram rules for the Green's functions are very simple. In the $2 n$-th order there are $3 n+1$ pairs of creation and annihilation operators. This leads to the factor: $[i \hbar]^{-2 n}[i \hbar]^{3 n+1}$. Canceling one factor $i \hbar$ leads to the following factor: $(-1)^{N_{F}}[i \hbar]^{n}|W|^{2 n}$ where $N_{F}$ is the number of closed fermion loops. With this rule the NCA self-energies (displayed in Fig. 2) are given by the equations

$$
\begin{aligned}
& \Sigma_{f}\left(\lambda ; t, t^{\prime}\right)=i \hbar|W|^{2} G_{b}\left(\lambda ; t, t^{\prime}\right) \tilde{D}_{A}\left(t, t^{\prime}\right), \\
& \Sigma_{b}\left(\lambda ; t, t^{\prime}\right)=-i \hbar N_{s}|W|^{2} G_{f}\left(\lambda ; t, t^{\prime}\right) \tilde{D}_{A}\left(t^{\prime}, t\right) .
\end{aligned}
$$

Here $N_{s}=2$ accounts for the spin degeneracy of singly occupied state for the $\operatorname{dot} S . G_{b}$ and $G_{f}$ are the psepdo particle Green's functions of boson and fermion, respectively. Using analytic continuation to the real-time axis 13 we find

$$
\begin{aligned}
& \Sigma_{f}^{>}\left(\lambda ; t, t^{\prime}\right)=|W|^{2} G_{b}^{>}\left(\lambda ; t, t^{\prime}\right) \tilde{D}_{A}^{>}\left(t, t^{\prime}\right), \\
& \Sigma_{f}^{<}\left(\lambda ; t, t^{\prime}\right)=-|W|^{2} G_{b}^{<}\left(\lambda ; t, t^{\prime}\right) \tilde{D}_{A}^{<}\left(t, t^{\prime}\right), \\
& \Sigma_{f}^{r}\left(\lambda ; t, t^{\prime}\right)=|W|^{2}\left[G_{b}^{r}\left(\lambda ; t, t^{\prime}\right) \tilde{D}_{A}^{>}\left(t, t^{\prime}\right)-G_{b}^{<}\left(\lambda ; t, t^{\prime}\right) \tilde{D}_{A}^{r}\left(t, t^{\prime}\right)\right], \\
& \Sigma_{b}^{>}\left(\lambda ; t, t^{\prime}\right)=N_{s}|W|^{2} G_{f}^{>}\left(\lambda ; t, t^{\prime}\right) \tilde{D}_{A}^{<}\left(t^{\prime}, t\right), \\
& \Sigma_{b}^{<}\left(\lambda ; t, t^{\prime}\right)=-N_{s}|W|^{2} G_{f}^{<}\left(\lambda ; t, t^{\prime}\right) \tilde{D}_{A}^{>}\left(t^{\prime}, t\right), \\
& \Sigma_{b}^{r}\left(\lambda ; t, t^{\prime}\right)=N_{s}|W|^{2}\left[G_{f}^{r}\left(\lambda ; t, t^{\prime}\right) \tilde{D}_{A}^{<}\left(t^{\prime}, t\right)+G_{f}^{<}\left(\lambda ; t, t^{\prime}\right) \tilde{D}_{A}^{a}\left(t^{\prime}, t\right)\right] .
\end{aligned}
$$

To give a concrete example of analytic continuation we consider the derivation of $\Sigma_{b}^{>}\left(\lambda ; t, t^{\prime}\right)$ :

$$
\frac{1}{i} \Sigma_{b}^{>}\left(\lambda ; t, t^{\prime}\right)=-i \hbar|W|^{2} \frac{1}{i} G_{f}^{>}\left(\lambda ; t, t^{\prime}\right) \times \frac{-1}{i} \tilde{D}_{A}^{<}\left(t^{\prime}, t\right) .
$$

Removing the common factors on both sides of the equation, we find the desired expression for $\Sigma_{b}^{>}\left(\lambda ; t, t^{\prime}\right)$.

After Fourier transform, we get the self-energy equations

$$
\begin{aligned}
& \Sigma_{f}^{>}(\lambda ; \omega)=|W|^{2} \int \frac{d \zeta}{2 \pi} G_{b}^{>}(\lambda ; \omega-\zeta) \tilde{D}_{A}^{>}(\zeta), \\
& \Sigma_{f}^{<}(\lambda ; \omega)=-|W|^{2} \int \frac{d \zeta}{2 \pi} G_{b}^{<}(\lambda ; \omega-\zeta) \tilde{D}_{A}^{<}(\zeta),
\end{aligned}
$$




$$
\begin{aligned}
& \Sigma_{f}^{r}(\lambda ; \omega)=|W|^{2} \int \frac{d \zeta}{2 \pi}\left[G_{b}^{r}(\lambda ; \omega-\zeta) \tilde{D}_{A}^{>}(\zeta)-G_{b}^{<}(\lambda ; \omega-\zeta) \tilde{D}_{A}^{r}(\zeta)\right], \\
& \Sigma_{b}^{>}(\lambda ; \omega)=N_{s}|W|^{2} \int \frac{d \zeta}{2 \pi} G_{f}^{>}(\lambda ; \omega+\zeta) \tilde{D}_{A}^{<}(\zeta), \\
& \Sigma_{b}^{<}(\lambda ; \omega)=-N_{s}|W|^{2} \int \frac{d \zeta}{2 \pi} G_{f}^{<}(\lambda ; \omega+\zeta) \tilde{D}_{A}^{>}(\zeta), \\
& \Sigma_{b}^{r}(\lambda ; \omega)=N_{s}|W|^{2} \int \frac{d \zeta}{2 \pi}\left[G_{f}^{r}(\lambda ; \omega+\zeta) \tilde{D}_{A}^{<}(\zeta)+G_{f}^{<}(\lambda ; \omega+\zeta) \tilde{D}_{A}^{a}(\zeta)\right] .
\end{aligned}
$$

The Green's function at the dot $S$ can be written down in terms of pseudo particle operators.

$$
i \hbar D_{S}\left(\lambda ; t, t^{\prime}\right)=<T d_{S \alpha}(t) d_{S \alpha}^{\dagger}\left(t^{\prime}\right)>=<T b^{\dagger}(t) f_{\alpha}(t) f_{\alpha}^{\dagger}\left(t^{\prime}\right) b\left(t^{\prime}\right)>.
$$

In the leading approximation the desired Green's function is

$$
D_{S}\left(\lambda ; t, t^{\prime}\right) \approx i \hbar G_{f}\left(\lambda ; t, t^{\prime}\right) G_{b}\left(\lambda ; t^{\prime}, t\right) .
$$

Analytic continuation to the real-time axis 13 leads to

$$
\begin{aligned}
& D_{S}^{>}\left(\lambda ; t, t^{\prime}\right)=-G_{f}^{>}\left(\lambda ; t, t^{\prime}\right) G_{b}^{<}\left(\lambda ; t^{\prime}, t\right), \\
& D_{S}^{<}\left(\lambda ; t, t^{\prime}\right)=G_{f}^{<}\left(\lambda ; t, t^{\prime}\right) G_{b}^{>}\left(\lambda ; t^{\prime}, t\right), \\
& D_{S}^{r}\left(\lambda ; t, t^{\prime}\right)=-G_{f}^{r}\left(\lambda ; t, t^{\prime}\right) G_{b}^{<}\left(\lambda ; t^{\prime}, t\right)-G_{f}^{<}\left(\lambda ; t, t^{\prime}\right) G_{b}^{a}\left(\lambda ; t^{\prime}, t\right) .
\end{aligned}
$$

After Fourier transform we get

$$
\begin{aligned}
& D_{S}^{>}(\lambda ; \omega)=-\int \frac{d \zeta}{2 \pi} G_{f}^{>}(\lambda ; \omega+\zeta) G_{b}^{<}(\lambda ; \zeta), \\
& D_{S}^{<}(\lambda ; \omega)=\int \frac{d \zeta}{2 \pi} G_{f}^{<}(\lambda ; \omega+\zeta) G_{b}^{>}(\lambda ; \zeta), \\
& D_{S}^{r}(\lambda ; \omega)=-\int \frac{d \zeta}{2 \pi}\left[G_{f}^{r}(\lambda ; \omega+\zeta) G_{b}^{<}(\lambda ; \zeta)+G_{f}^{<}(\lambda ; \omega+\zeta) G_{b}^{a}(\lambda ; \zeta)\right] .
\end{aligned}
$$

From the projection to the physical Hilbert space $Q=1$ of the Green's function at the $\operatorname{dot} S$, we find the projection rules for the pseudo particle Green's functions.

$$
\begin{aligned}
D_{S}^{r}(\omega) & =\frac{1}{Z_{S}} \lim _{\lambda \rightarrow \infty} e^{\beta \lambda} D_{S}^{r}(\lambda ; \omega) \\
& =-\frac{1}{Z_{S}} \lim _{\lambda \rightarrow \infty} e^{\beta \lambda} \int \frac{d \zeta}{2 \pi}\left[G_{f}^{r}(\lambda ; \omega+\zeta+\lambda) G_{b}^{<}(\lambda ; \zeta+\lambda)+G_{f}^{<}(\lambda ; \omega+\zeta+\lambda) G_{b}^{a}(\lambda ; \zeta+\lambda)\right] \\
& =-\frac{1}{Z_{S}} \int \frac{d \zeta}{2 \pi}\left[G_{f}^{r}(\omega+\zeta) G_{b}^{<}(\zeta)+G_{f}^{<}(\omega+\zeta) G_{b}^{a}(\zeta)\right], \\
Z_{S} & =\int \frac{d \omega}{2 \pi}\left[N_{s} G_{f}^{<}(\omega)-G_{b}^{<}(\omega)\right] .
\end{aligned}
$$

Since the pseudo particle Green's functions are centered at $\omega=E_{S}+\lambda$ (pseudo fermion) and at $\omega=\lambda$ (slave boson), we have to shift the integration variable before taking the limit: $\lambda \rightarrow \infty$. For $D_{S}^{r}(\omega)$ to be well-defined the limiting pseudo particle Green's functions should be defined as

$$
\begin{aligned}
& G_{b, f}^{r}(\omega) \equiv \lim _{\lambda \rightarrow \infty} G_{b, f}^{r}(\lambda ; \omega+\lambda), \\
& G_{b, f}^{<}(\omega) \equiv \lim _{\lambda \rightarrow \infty} e^{\beta \lambda} G_{b, f}^{<}(\lambda ; \omega+\lambda) .
\end{aligned}
$$

The second equation means that $\lim _{\lambda \rightarrow \infty} G_{b, f}^{<}(\lambda ; \omega+\lambda)=0$. Applying the projection procedure to the lesser and greater Green's functions of the dot $S$ we find the additional projection rules for the pseudo particle Green's functions:

$$
G_{b, f}^{>}(\omega) \equiv \lim _{\lambda \rightarrow \infty} G_{b, f}^{>}(\lambda ; \omega+\lambda)
$$


Accordingly the lesser and greater Green's functions of the $\operatorname{dot} S$ are given by the equations

$$
\begin{aligned}
& D_{S}^{<}(\omega)=\frac{1}{Z_{S}} \int \frac{d \zeta}{2 \pi} G_{f}^{<}(\omega+\zeta) G_{b}^{>}(\zeta), \\
& D_{S}^{>}(\omega)=-\frac{1}{Z_{S}} \int \frac{d \zeta}{2 \pi} G_{f}^{>}(\omega+\zeta) G_{b}^{<}(\zeta) .
\end{aligned}
$$

Applying the projection procedure to the physical Green's function $D_{S}$ at the $\operatorname{dot} S$ we obtained the projection rules for the pseudo particle Green's functions.

We are now in a position to find the self-energy equations projected to the physical Hilbert space, $Q=1$, for the pseudo particle Green's functions.

$$
\begin{aligned}
& \Sigma_{b, f}^{r,>}(\omega)=\lim _{\lambda \rightarrow \infty} \Sigma_{b, f}^{r,>}(\lambda ; \omega+\lambda), \\
& \Sigma_{b, f}^{<}(\omega)=\lim _{\lambda \rightarrow \infty} e^{\beta \lambda} \Sigma_{b, f}^{<}(\lambda ; \omega+\lambda) .
\end{aligned}
$$

By comparing the self-energies we can see the identities: $G_{b, f}^{r}(\omega)=G_{b, f}^{>}(\omega)$.

$$
\begin{aligned}
& \Sigma_{f}^{<}(\omega)=-|W|^{2} \int \frac{d \zeta}{2 \pi} G_{b}^{<}(\omega-\zeta) \tilde{D}_{A}^{<}(\zeta), \\
& \Sigma_{f}^{r}(\omega)=|W|^{2} \int \frac{d \zeta}{2 \pi} G_{b}^{r}(\omega-\zeta) \tilde{D}_{A}^{>}(\zeta), \\
& \Sigma_{b}^{<}(\omega)=-N_{s}|W|^{2} \int \frac{d \zeta}{2 \pi} G_{f}^{<}(\omega+\zeta) \tilde{D}_{A}^{>}(\zeta), \\
& \Sigma_{b}^{r}(\omega)=N_{s}|W|^{2} \int \frac{d \zeta}{2 \pi} G_{f}^{r}(\omega+\zeta) \tilde{D}_{A}^{<}(\zeta) .
\end{aligned}
$$

These are the NCA self-energy equations out of equilibrium. Now inserting the following equations

$$
\begin{aligned}
& \tilde{D}_{A}^{<}(\omega)=\left|\tilde{D}_{A}^{r}(\omega)\right|^{2}\left[2 \Gamma_{L}(\omega) f_{L}(\omega)+2 \Gamma_{R}(\omega) f_{R}(\omega)\right], \\
& \tilde{D}_{A}^{>}(\omega)=\left|\tilde{D}_{A}^{r}(\omega)\right|^{2}\left[2 \Gamma_{L}(\omega) f_{L}(-\omega)+2 \Gamma_{R}(\omega) f_{R}(-\omega)\right],
\end{aligned}
$$

we find the final version of the NCA equations

$$
\begin{aligned}
& \Sigma_{f}^{<}(\omega)=-\sum_{p=L, R} \int \frac{d \zeta}{\pi}|W|^{2}\left|\tilde{D}_{A}^{r}(\zeta)\right|^{2} \Gamma_{p}(\zeta) f_{p}(\zeta) G_{b}^{<}(\omega-\zeta), \\
& \Sigma_{f}^{r}(\omega)=\sum_{p=L, R} \int \frac{d \zeta}{\pi}|W|^{2}\left|\tilde{D}_{A}^{r}(\zeta)\right|^{2} \Gamma_{p}(\zeta) f_{p}(-\zeta) G_{b}^{r}(\omega-\zeta), \\
& \Sigma_{b}^{<}(\omega)=-N_{s} \sum_{p=L, R} \int \frac{d \zeta}{\pi}|W|^{2}\left|\tilde{D}_{A}^{r}(\zeta)\right|^{2} \Gamma_{p}(\zeta) f_{p}(-\zeta) G_{f}^{<}(\omega+\zeta), \\
& \Sigma_{b}^{r}(\omega)=N_{s} \sum_{p=L, R} \int \frac{d \zeta}{\pi}|W|^{2}\left|\tilde{D}_{A}^{r}(\zeta)\right|^{2} \Gamma_{p}(\zeta) f_{p}(\zeta) G_{f}^{r}(\omega+\zeta) .
\end{aligned}
$$

The effective Anderson hybridization for a side-connected dot $S$ is given by $\Gamma_{\text {eff }}(\omega)=|W|^{2}\left|\tilde{D}_{A}^{r}(\omega)\right|^{2}\left[\Gamma_{L}(\omega)+\Gamma_{R}(\omega)\right]$. Although the $\operatorname{dot} S$ is not directly coupled to the external electrodes it can experience the bias potential difference via the active $\operatorname{dot} A$.

\section{NUMERICAL RESULTS}

For the numerical calculations, we assume the constant energy-independent tunneling matrix between the active quantum $\operatorname{dot} A$ and two electrodes. The left and right electrodes are assumed to be described by the same Lorentzian density of states (DOS). The self-energies due to the tunneling into the electrodes are found in a closed form in this case. The auxillary Green's function for the active $\operatorname{dot} A\left(\tilde{D}_{A}^{r}\right)$ and the effective Anderson hybridization function for the side-connected $\operatorname{dot} S\left(\Gamma_{\text {eff }}(\omega)=\tilde{\Gamma}_{L}(\omega)+\tilde{\Gamma}_{R}(\omega)\right)$ are given by the equations 


$$
\begin{aligned}
\tilde{D}_{A}^{r}(\omega) & =\frac{1}{\hbar \omega-E_{A}-\Gamma \times \frac{D}{\hbar \omega+i D}} \\
\tilde{\Gamma}_{p}(\omega) & =\Gamma_{p}|W|^{2} \frac{D^{2}}{\left[\hbar \omega\left(\hbar \omega-E_{A}\right)-\Gamma D\right]^{2}+\left(\hbar \omega-E_{A}\right)^{2} D^{2}}
\end{aligned}
$$

Here $D$ is the conduction band width of the Lorentzian DOS. The tunneling rate of an active $\operatorname{dot} A$ into the electrodes $\Gamma=\Gamma_{L}+\Gamma_{R}$ or the effective band width of Anderson hybridization $\Gamma_{\text {eff }}(\omega)$ will be taken as an energy unit in our numerical works. For this model the Kondo temperature at zero source-drain bias voltage can be estimated within an order of 1 by the equation

$$
T_{K}=\Gamma \times \exp \left(-\frac{\pi\left|E_{S}\right|}{2 \Gamma_{\mathrm{eff}}}\right)
$$

Here $\Gamma_{\text {eff }}=|W|^{2} / \Gamma$ is the effective Anderson hybridization at $\omega=0$ and $E_{A}=0$.

Self-energies: The self-energy $\Sigma_{S}$ of a side-connected $\operatorname{dot} S$ is obtained from the Green's function $D_{S}$ which is calculated from equation (3.16), using the relation $\Sigma_{S}^{r}=\left[D_{S}^{r}\right]^{-1}-\left[D_{S 0}^{r}\right]^{-1}$. The imaginary part of $\Sigma_{S}^{r}$ is displayed in Fig 3. Displayed results are corrected to satisfy the causality relation for the Greep's function at the active $\operatorname{dot} A$. This correction procedure is explained in the Appendix $\mathrm{C}$ and see also the references 18 . With decreasing temperature a sharp feature develops near $\omega=0$, which is a sign of Kondo resonance peak. When a finite source-to-drain bias voltage is applied the sharp structure near $\omega=0$ gets smooth and split into two at $\omega= \pm e V / 2$.

Spectral functions: The spectral functions are calculated from the imaginary part of Green's functions

$$
A_{i}(\omega)=-\frac{1}{\pi} \operatorname{Im} D_{i}^{r}(\omega), \quad i=A, S,
$$

and displayed in Figs. tand 5 . The spectral function $A_{S}(\omega)$ at a side-connected dot $S$ develops the Kondo resonance peak near $\omega=0$ with decreasing temperature below $T<T_{K}$. The Green's function at the active $\operatorname{dot} A$ is renormalized according to the equation (2.22). As the side-connected dot $S$ develops the Kondo resonance, the spectral function $A_{A}(\omega)$ at the active $\operatorname{dot} A$ is depleted near $\omega=0$. The spectral depletion can be understood as the destructive interference between the direct path and the indirect path (Fano interference). The spectral depletion leads to the suppression of the current flowing through the $\operatorname{dot} A$. The width of depleted region is given by the Kondo temperature (4.3) of the $\operatorname{dot} S$. When a finite value of source-to-drain bias voltage is applied, the peak in $A_{S}(\omega)$ and the dip in $A_{A}(\omega)$ near $\omega=0$ get splits into two at $\omega= \pm e V / 2$. The two Kondergsonance peaks in $A_{S}(\omega)$ and dips in $A_{A}(\omega)$ become pinned at the Fermi energies of the left and right electrodes.16.17

Since the NCA underestimates one-body contribution of the self-energy $\Sigma_{S}$ (side-connected dot) from tunneling into the effective continuum band, our NCA calculation of the spectral function at the active $\operatorname{dot} A$ shows the negative spectral weight near the depleted region (violation of the causality relation). This is a purely artifact of the NCA. Since the NCA is based on the expansion in powers of the Anderson hybridization (in our case $W$ ) and includes only a subset of diagrams up to the infinite order in $W$ without vertex corrections, the underestimation is expected. Though the NCA underestimates the one-body contribution of the self-energy, its dependence on the energy and temperature turns out to be correct close to $\omega=0$ and at $T>0 \mathrm{~K}$. The NCA leads to the pathological results at and near $T=0 \mathrm{~K} .14$ From the Fermi-liquid relation it can be shown that the spectral function of the active $\operatorname{dot} A$ is zero at $\omega=0$ at zero temperature. According to the Fermi-liquid theory, the magnetic ion's spectral function $\left(A_{S}(\omega)\right.$ in our case) can be related to the phase shift of conduction electrons (electrons-at the active $\operatorname{dot} A$ in our case) at the Fermi energy. The phase shift is determined by the Langreth-Friedel sum rule 2 , $n_{S}=2 \frac{\delta}{\pi}$, where the numerical factor 2 accounts for two possible spin directions.

$$
A_{S}(0)=\frac{1}{\pi \Gamma_{\mathrm{eff}}} \sin ^{2} \frac{\pi n_{S}}{2}
$$

where $\Gamma_{\text {eff }}=\frac{|W|^{2}}{\Gamma}$ is the effective Anderson hybridization for a side-connected $\operatorname{dot} S$ at $\omega=0$. With complete screening $n_{S}$ is equal to 1 . Inserting the Fermi-liquid relation into the equation (2.22) it can be shown that the spectral function of the active $\operatorname{dot} A$ vanishes at $\omega=0$, or $A_{A}(0)=0$.

To compensate the NCA's underestimation of the self-energy we corrected the self-energy by adding a term proportional to one-body self-energy $|W|^{2} \tilde{D}_{A}^{r}(\omega)$ to it such that the spectral function $A_{A}$ of the active dot remains non-negative definite and $A_{A}(0) \rightarrow 0$ with the temperature approaching zero. Since the contribution to the selfenergy coming from the hybridization with the effective continuum band is of the one-body nature and temperatureindependent, this correction proedure is not a bad idea. 
Linear response conductance: The linear response conductance $\sigma(T)$ is calculated by differentiating the equation (2.21a) with respect to the source-drain bias voltage at $V=0$.

$$
\sigma(T)=\frac{2 e^{2}}{h} \int d \epsilon T(\epsilon)\left[-\frac{\partial f(\epsilon)}{\partial \epsilon}\right] .
$$

As shown in Fig. 6, $\sigma(T)$ is suppressed with decreasing temperature. The conductance shows a logarithmic dependence on temperature near the Kondo temperature. From Fermi-liquid theory it can be expected that the conductance is completely suppressed at zero temperature. The electron flow is blocked by the destructive inteference between two different paths: the direct path $(L \rightarrow A \rightarrow R)$ and the indirect one $(L \rightarrow A \rightarrow S \rightarrow A \rightarrow R)$.

This suppressed behavior of $\sigma(T)$ at low temperatures is another example of zero-bias anomalies (ZBA) observed in several mesoscopic devices. Scattering of conduction electrons off the Kondo impurities is one of scenarios explaining ZBA's. Depending on the topology of mesoscopic devices and Kondo impurites, the ZBA can lead to the enhanced or suppressed conductance at low temperature.

As expected from the Kondo effect, the linear response conductance $\sigma(T)$ shows the scaling behavior over a wide range of temperature. Several curves of $\sigma(T)$ for different sets of model parameters (differing Kondo temperature) collapse onto one curve as shown in Fig. 6 .

Differential conductance: The current flowing from the left to right electrode is calculated using the equation (2.21a). Differential conductance $G(T, V)=d I / d V$ is obtained by differentiating the current with respect to a finite sourcedrain bias voltage. Differential conductance is displayed in Fig. 7. With decreasing temperature $d I / d V$ is suppressed near zero source-drain bias voltage. The pseudo gap behavior of the spectral function at the active quantum dot $A$ explains this $I-V$ characteristics.

\section{CONCLUSION}

In this work we studied the transport properties of parallel double quantum dots when only the active dot is coupled to the two external electrodes and the other dot is side-connected to the active dot. When the active dot lies in the conductance peak region and the side-connected dot in the Coulomb blockade region, the current flow through the active dot is suppressed due to the Kondo scattering off the effective spin in the side-connected dot. Suppressed conductance at low temperature and near zero bias voltage can be understood in terms of Fano interference between

two paths: direct path of left electrode - active dot - right electrode and indirect path of left electrode - active dot - side-coneccted dot - active-dot - right electrode. Our study suggests a possibility that the current flowing through one quantum dot can be controlled experimentally by an additional side-connected quantum dot.

\section{ACKNOWLEDGMENTS}

This work is supported in part by the BK21 project and in part by the National Science Foundation under Grant No. DMR 9357474.

\section{APPENDIX A: NON-INTERACTING CASE: $U_{A}=U_{S}=0$}

When $U_{A}=U_{S}=0$, both active and side-connected dots become resonant levels. The current and its noise can be obtained analytically in a closed form. The self-energies of the $\operatorname{dots} A$ and $S$ are

$$
\begin{aligned}
& \Sigma_{A}\left(t, t^{\prime}\right)=\Sigma_{c}\left(t, t^{\prime}\right)+|W|^{2} D_{S 0}\left(t, t^{\prime}\right), \\
& \Sigma_{S}\left(t, t^{\prime}\right)=|W|^{2} \tilde{D}_{A}\left(t, t^{\prime}\right) .
\end{aligned}
$$

Here the one-body self-energy $\Sigma_{c}$ is given by the equation

$$
\Sigma_{c}\left(t, t^{\prime}\right)=\frac{1}{V} \sum_{p \vec{k}}\left|V_{p}(\vec{k})\right|^{2} G_{c p}\left(\vec{k} ; t, t^{\prime}\right),
$$

and the auxillary Green's functions $\tilde{D}_{A}$ and $\tilde{D}_{S}$ are given by the equations 


$$
\begin{aligned}
& \tilde{D}_{A}\left(t, t^{\prime}\right)=D_{A 0}\left(t, t^{\prime}\right)+\int_{C} d t_{1} \int_{C} d t_{2} \tilde{D}_{A}\left(t, t_{1}\right) \Sigma_{c}\left(t_{1}, t_{2}\right) D_{A 0}\left(t_{2}, t^{\prime}\right), \\
& \tilde{D}_{S}\left(t, t^{\prime}\right)=D_{S 0}\left(t, t^{\prime}\right) .
\end{aligned}
$$

The self-energy of the auxillary Green's function $\tilde{D}_{A}$ is given by $\tilde{\Sigma}_{A}\left(t, t^{\prime}\right)=\Sigma_{c}\left(t, t^{\prime}\right)$.

The retarded Green's functions can be readily obtained

$$
\begin{aligned}
D_{A}^{r}(\omega) & =\frac{\hbar \omega-E_{S}}{\left[\hbar \omega-E_{S}\right] \times\left[\hbar \omega-E_{A}-\Sigma_{c}^{r}(\omega)\right]-|W|^{2}}, \\
D_{S}^{r}(\omega) & =\frac{\hbar \omega-E_{A}-\Sigma_{c}^{r}(\omega)}{\left[\hbar \omega-E_{S}\right] \times\left[\hbar \omega-E_{A}-\Sigma_{c}^{r}(\omega)\right]-|W|^{2}} .
\end{aligned}
$$

Due to the Fano interference the spectral function of the active $\operatorname{dot} A$ vanishes at $\omega=E_{S}$. On the other hand the spectral function of the side-connected $\operatorname{dot} S$ is peaked at $\omega=E_{S}$. The nonequilibrium distribution function of the $\operatorname{dot} S$ is also readily calculated

$$
\begin{aligned}
\tilde{\Sigma}_{A}^{<}(\omega) & =2 \Gamma_{L}(\omega) f_{L}(\omega)+2 \Gamma_{R}(\omega) f_{R}(\omega), \\
-\operatorname{Im} \tilde{\Sigma}_{A}^{r}(\omega) & =\Gamma_{L}(\omega)+\Gamma_{R}(\omega) \\
f_{\mathrm{eff}}(\omega) & =\frac{\tilde{\Sigma}_{A}^{<}(\omega)}{-2 \operatorname{Im} \tilde{\Sigma}_{A}^{r}(\omega)}=\frac{\Gamma_{L}(\omega) f_{L}(\omega)+\Gamma_{R}(\omega) f_{R}(\omega)}{\Gamma_{L}(\omega)+\Gamma_{R}(\omega)} .
\end{aligned}
$$

The full self-energies of the active $\operatorname{dot} A$ are

$$
\begin{aligned}
\Sigma_{A}^{<}(\omega) & =2 \Gamma_{L}(\omega) f_{L}(\omega)+2 \Gamma_{R}(\omega) f_{R}(\omega)+|W|^{2} \tilde{D}_{S}^{<}(\omega), \\
\Sigma_{A}^{r}(\omega)-\Sigma_{A}^{a}(\omega) & =-2 i\left[\Gamma_{L}(\omega)+\Gamma_{R}(\omega)\right]+|W|^{2}\left[\tilde{D}_{S}^{r}(\omega)-\tilde{D}_{S}^{a}(\omega)\right] .
\end{aligned}
$$

To determine $\tilde{D}_{S}^{<}(\omega)$, we use the condition $I_{S}=0$ (the number of electrons in the $\operatorname{dot} S$ remains the same).

$$
\tilde{D}_{S}^{<}(\omega)=2 \pi \frac{\Gamma_{L}(\omega) f_{L}(\omega)+\Gamma_{R}(\omega) f_{R}(\omega)}{\Gamma_{L}(\omega)+\Gamma_{R}(\omega)} \tilde{A}_{S}(\omega)
$$

Here $\tilde{A}_{S}(\omega)=\delta\left(\hbar \omega-E_{S}\right)$.

Inserting all the equations into the expression of the current (2.7), we find the current flowing from the left electrode to the right one.

$$
\begin{aligned}
I_{L}=e \sum_{\alpha} \int \frac{d \omega}{2 \pi}\left\{4 \Gamma_{L}(\omega) \Gamma_{R}(\omega)\left|D_{A}^{r}(\omega)\right|^{2}\left[f_{R}(\omega)-f_{L}(\omega)\right]\right. \\
\left.+2|W|^{2} \Gamma_{L}(\omega)\left|D_{A}^{r}(\omega)\right|^{2}\left[\tilde{D}_{S}^{<}(\omega)-2 \pi f_{L}(\omega) \tilde{A}_{S}(\omega)\right]\right\} \\
=\frac{e}{h} \sum_{\alpha} \int d \epsilon T(\epsilon)\left[f_{R}(\epsilon)-f_{L}(\epsilon)\right] .
\end{aligned}
$$

The transmission spectral function $T(\epsilon)$ is given by the equation

$$
T(\epsilon)=4 \Gamma_{L}(\epsilon) \Gamma_{R}(\epsilon)\left|D_{A}^{r}(\epsilon)\right|^{2}\left[1+\frac{\pi|W|^{2} \tilde{A}_{S}(\epsilon)}{\Gamma_{L}(\epsilon)+\Gamma_{R}(\epsilon)}\right]=\frac{4 \pi \Gamma_{L}(\epsilon) \Gamma_{R}(\epsilon)}{\Gamma_{L}(\epsilon)+\Gamma_{R}(\epsilon)} A_{A}(\epsilon) .
$$

The current noise, defined as the current-current correlation function, can be obtained in a closed form in the noninteracting case.

$$
S\left(t, t^{\prime}\right)=<\delta I(t) \delta I\left(t^{\prime}\right)>+<\delta I\left(t^{\prime}\right) \delta I(t)>.
$$

Here $\delta I=I-<I>$. Defining the current Green's function by

$$
i \hbar G_{I I}\left(t, t^{\prime}\right)=<T \delta I(t) \delta I\left(t^{\prime}\right)>
$$


the current-current correlation function can be written as $S\left(t, t^{\prime}\right)=\hbar G_{I I}^{>}\left(t, t^{\prime}\right)+\hbar G_{I I}^{>}\left(t^{\prime}, t\right)$. Six diagrams are contributing to the current noise and the static current noise $S_{0}=S(\omega=0)$ is given by the well-known formula.20

$$
\begin{aligned}
S_{0}= & \frac{4 e^{2}}{h} \int d \epsilon T(\epsilon)\left\{f_{L}(\epsilon)\left[1-f_{L}(\epsilon)\right]+f_{R}(\epsilon)\left[1-f_{R}(\epsilon)\right]\right\} \\
& +\frac{4 e^{2}}{h} \int d \epsilon T(\epsilon)[1-T(\epsilon)]\left\{f_{L}(\epsilon)-f_{R}(\epsilon)\right\}^{2} .
\end{aligned}
$$

The first line is the thermal Johnson noise and the second line is called the shot noise coming from a finite source-drain bias voltage.

Typical results at $T=0 \mathrm{~K}$ are displayed in Fig. 8 for the transmission coefficient $T(\epsilon)$ and the Fano factor $F=$ $S_{0} / 2 e I$. Due to the Fano interference the transmission coefficient is completely suppressed at $\omega=E_{S}$, the energy level of the side-connected dot. This structure leads to a dip in the Fano factor. The Fano factor $F$ takes the following values at $V=0$ and $V \rightarrow \infty$.

$$
\begin{aligned}
F(V=0) & =1-\frac{4 \Gamma_{L} \Gamma_{R}}{\left(\Gamma_{L}+\Gamma_{R}\right)^{2}} \frac{E_{S}^{2}}{E_{S}^{2}+\left(E_{A} E_{S}-W^{2}\right)^{2} / \Gamma^{2}}, \\
F(V \rightarrow \infty) & =1-\frac{2 \Gamma_{L} \Gamma_{R}}{\left(\Gamma_{L}+\Gamma_{R}\right)^{2}} .
\end{aligned}
$$

Here $\Gamma=\Gamma_{L}+\Gamma_{R}$. The limiting value of $F$ at $e V \gg \Gamma$ is solely determined by the tunneling rates, $\Gamma_{L}$ and $\Gamma_{R}$.

\section{APPENDIX B: INTERACTING CASE: $U_{A} \neq 0$ AND $U_{S} \neq 0$}

When both dots $A$ and $S$ are strongly correlated, the model system becomes highly non-trivial due to many-body effects. In this section we derive the effective spin exchange interaction when both dots lie in the Kondo limit or in the absence of charge fluctuations. Our model space $\mathcal{M}$ and its orthogonal space $\overline{\mathcal{M}}$ are then given by

$$
\begin{aligned}
& \mathcal{M}=|A \alpha>\otimes| S \beta>=\mid A \alpha ; S \beta>, \quad \alpha, \beta=\uparrow, \downarrow, \\
& \overline{\mathcal{M}}=|A \alpha ; S \rho>,| A \rho ; S \alpha>, \mid A \rho ; S \rho^{\prime}>, \quad \alpha, \beta=\uparrow, \downarrow ; \quad \rho, \rho^{\prime}= \pm .
\end{aligned}
$$

Here $\alpha$ and $\beta$ represent the spin up or down state, while $\rho$ and $\rho^{\prime}$ the isospin state:

$$
\left|i+>=d_{i \uparrow}^{\dagger} d_{i \downarrow}^{\dagger}\right| 0>, \quad|i->=| 0>, \quad i=A, S
$$

where $\mid 0>$ means the empty state of a quantum dot.

To find the effective spin-exchange Hamiltonian we need to evaluate the following matrix elements

$$
\begin{aligned}
<A \alpha ; S \beta\left|H_{1}\right| A \mu ; S \rho> & =0 \\
<A \alpha ; S \beta\left|H_{1}\right| A \rho ; S \mu> & =\delta_{\beta, \mu}<A \alpha\left|H_{1}\right| A \rho> \\
& =\delta_{\beta, \mu} \times\left\{\sum_{p \vec{k}} V_{p}^{*}(\vec{k}) c_{p \vec{k} \alpha} \delta_{\rho,-}+(-1)^{\alpha-1 / 2} \sum_{p \vec{k}} V_{p}(\vec{k}) c_{p \vec{k} \bar{\alpha}}^{\dagger} \delta_{\rho,+}\right\} \\
<A \alpha ; S \beta\left|H_{1}\right| A \rho ; S \rho^{\prime}> & =(-1)^{\alpha-1 / 2} W \delta_{\alpha+\beta, 0} \delta_{\rho+\rho^{\prime}, 0} .
\end{aligned}
$$

When the charge fluctuations can be neglected we find the effective spin-exchange Hamiltonian

$$
\begin{aligned}
\tilde{H}_{1} & =\sum_{p \vec{k} \alpha} \sum_{p^{\prime} \vec{k}^{\prime} \beta} J_{p p^{\prime}}\left(\vec{k}, \vec{k}^{\prime}\right) c_{p \vec{k} \alpha}^{\dagger} \frac{1}{2} \vec{\sigma}_{\alpha \beta} c_{p^{\prime} \vec{k}^{\prime} \beta} \cdot \vec{S}_{A}+J_{A S}\left(\vec{S}_{A} \cdot \vec{S}_{S}-\frac{1}{4}\right), \\
J_{p p^{\prime}}\left(\vec{k}, \vec{k}^{\prime}\right) & =2 V_{p}(\vec{k}) V_{p^{\prime}}^{*}\left(\vec{k}^{\prime}\right)\left[\frac{1}{-E_{A}}+\frac{1}{E_{A}+U_{A}}\right], \\
J_{D} & =2|W|^{2}\left[\frac{1}{E_{A}+U_{A}-E_{S}}+\frac{1}{E_{S}+U_{S}-E_{A}}\right] .
\end{aligned}
$$

Electrons are scattered off an effective spin $\vec{S}_{A}$ at the active dot and can flow from one electrode to the other. Since $\vec{S}_{A}$ is coupled antiferromagnetically to $\vec{S}_{S}$, the occurence of enhanced Kondo scattering at low temperature depends 
on the magnitude of antiferromagnetic coupling $J_{A S}$ relative to, e.g., the Kondo temperature. When $J_{A S} \gg T_{K}$, a spin-singlet formation between two dots is favored over the many-body Kondo singlet state and the enhanced Kondo scattering is not expected. On the other hand when $T_{K} \gg J_{A S}$, a Kondo singlet state is expected to be favored over a spin-singlet state between two dots and the conductance will be enhanced due to the Kondo scattering at low temperature. Detailed study of this model in equilibrium is in progress using the Wilson's numerical renormalization and will be published elsewhere.

\section{APPENDIX C: FERMI-LIQUID RELATIONS}

In this section we discuss the correction of pathological behavior of NCA near $\omega=0$. The Green's function of the $\operatorname{dot} A$ is related to that at the $\operatorname{dot} S$ by the equation $(2.22)$. The causality is seldomly violated when the number of channels is larger than two. On the other hand the causality is violated for the case of one-channel near $\omega=0$ at temperatures below the Kondo temperature $T_{K}$. Recently developed conserving $t$-matrix approximation (CTMA) 11 may have a chance to overcome this pathology. In this paper we are going to remedy this pathology of the NCA by adding one-body self-energy correction to the self-energy $\Sigma_{S}(\omega)$ to satisfy the Fermi liquid relation. The self-energy for the $\operatorname{dot} S$ can be written as a sum of two contributions

$$
\Sigma_{S}^{r}(\omega)=\Sigma_{\mathrm{hyb}}^{r}(\omega)+\Sigma_{U}^{r}(\omega), \quad \Sigma_{\mathrm{hyb}}^{r}(\omega)=|W|^{2} \tilde{D}^{r}(\omega)
$$

The self-energy $\Sigma_{N C A}^{r}(\omega)$ calculated from the NCA is known to show the correct energy and temperature dependence near $\omega=0$ and $T=0$. (Temperature must be higher than the pathogical temperature estimated in, e.g., the references 14 .) We assume that $\Sigma_{U}(\omega)$ is well-captured in the NCA. Since $-\operatorname{Im} \Sigma_{U}^{r}(\omega) \propto \omega^{2}+\left[\pi k_{B} T\right]^{2}$ for $\omega, T<T_{K^{\prime}}$ (look at the references2 2 ), we add the correction term to $\Sigma_{N C A}$ in order to make $D_{A}^{r}(\omega)$ obey the causality relation.18

$$
\Sigma_{S}^{r}(\omega) \approx \Sigma_{N C A}^{r}(\omega)+\left(|W|^{2}-W_{N C A}^{2}\right) \tilde{D}_{A}^{r}(\omega)
$$

Here $W_{N C A}^{2}$ is determined numerically from the calculated $\Sigma_{N C A}^{r}(\omega)$ at the lowest possible temperature.

$$
\frac{W_{N C A}^{2}}{\Gamma} \equiv-\operatorname{Im} \Sigma_{N C A}^{r}(0)
$$

${ }^{1}$ L.I. Glazman and M.E. Raikh, JETP Lett. 47, 452 (1988); T.K. Ng and P.A. Lee, Phys. Rev. Lett. 61, 1768 (1988).

${ }^{2}$ D. Goldhaber-Gordon, H. Shtrikman, D. Malahu, D. Abusch-Magder, U. Meirav, and M.A. Kastner, Nature 391,156 (1998).

${ }^{3}$ S.M. Cronenwett, T.H. Oosterkamp, and L.P. Kouwenhoven, Science 281, 540 (1998).

4 J. Nygard, D.H. Cobden, and P.E. Lindelof, Nature 408, 342 (2000).

${ }^{5}$ M. Büttiker and C.A. Stafford, Phys. Rev. Lett. 76, 495 (1996); V. Ferrari, et. al., Phys. Rev. Lett. 82, 5088 (1999).

${ }^{6}$ U. Gerland, J. von Delft, T.A. Costi, and Y. Oreg, Phys. Rev. Lett. 84, 3710 (2000).

${ }^{7}$ W.G. van der Wiel, S. De Franceschi, T. Fujisawa, J.M. Elzerman, S. Tarucha, and L.P. Kouwenhoven, Science 289, 2105 (2000).

${ }^{8}$ R. Aguado and D.C. Langreth, Phys. Rev. Lett. 85, 1946 (2000); C.A. Büsser, E.V. Anda, A.L. Lima, M.A. Davidovich, and G. Chiappe, Phys. Rev. B 62, 9907 (2000); W. Izumida and O. Sakai, Phys. Rev. B 62, 10260 (2000).

${ }^{9}$ S. Sasaki, S. De Franceschi, J.M. Elzerman, W.G. van der Wiel, M. Eto, S. Tarucha, and L.P. Kouwenhoven, Nature 405, 764 (2000).

${ }^{10}$ M. Eto and Yu.V. Nazarov, Phys. Rev. Lett. 85, 1306 (2000); M. Pustilnik and L.I. Glazman, Phys. Rev. Lett. 85, 2993 (2000).

${ }^{11}$ J.R. Schrieffer and P.A. Wolff, Phys. Rev. 149, 491 (1966).

${ }^{12}$ L.V. Keldysh, Zh. Éksp. Teor. Fiz. 47, 1515 (1964). [Sov. Phys. JETP 20, 1018 (1965)]; Y. Meir and N. S. Wingreen, Phys. Rev. Lett. 68, 2512 (1992).

13 D.C. Langreth, 1976, in Linear and Nonlinear Electron Transport in Solids, Vol.17 of NATO Advanced Study Institute, Series B: Physicsi, edited by J.T. Devreese and V.E. van Doren (Plenum, New York, 1976), p. 3.

14 N.E. Bickers, Rev. Mod. Phys. 59, 845-939 (1987); N.E. Bickers, D.L. Cox, and J.W. Wilkins, Phys. Rev. B 36, 2036 (1987).

15 T.-S. Kim and D.L. Cox, Phys. Rev. B 55, 12594 (1997). 
${ }^{16}$ Y. Meir, N. S. Wingreen, and P. A. Lee, Phys. Rev. Lett. 70, 2601 (1993); N.S. Wingreen and Y. Meir, Phys. Rev. B 49, 11040 (1994).

${ }^{17}$ M.H. Hettler, J. Kroha, and S. Hershfield, Phys. Rev. Lett. 73, 1967 (1994).

18 D.L. Cox and N. Grewe, Z. Phys. B 71, 321 (1988); E. Kim and D.L. Cox, Phys. Rev. B 58, 3313 (1998).

19 D.C. Langreth, Phys. Rev. 150, 516 (1966).

${ }^{20}$ For instance, look at the review article, Ya. M. Blanter and M. Büttiker, Physics Reports 336, 1 (2000).

${ }^{21}$ J. Kroha, P. Wölfle, and T.A. Costi, Phys. Rev. Lett. 79, 261 (1997).

${ }^{22}$ K. Yamada, Prog. Theor. Phys. 53, 970 (1975); Prog. Theor. Phys. 54, 316 (1975). 


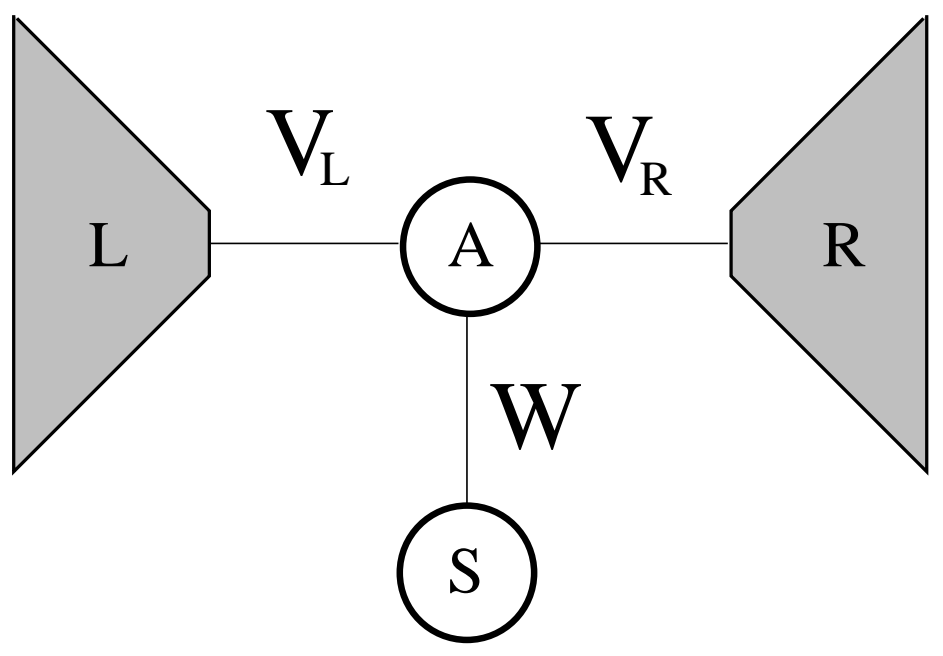

FIG. 1. Schematic display of parallel double quantum dots. The active quantum dot $A$ is connected to two left and right electrodes and the other $\operatorname{dot} S$ is side-connected to the $\operatorname{dot} A$. A side-connected dot $S$ provides an additional current path or acts as scattering center of electrons passing through the $\operatorname{dot} A$.

(a)

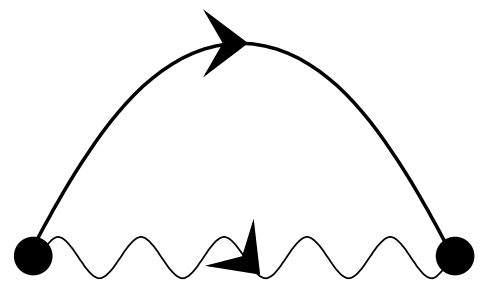

(b)

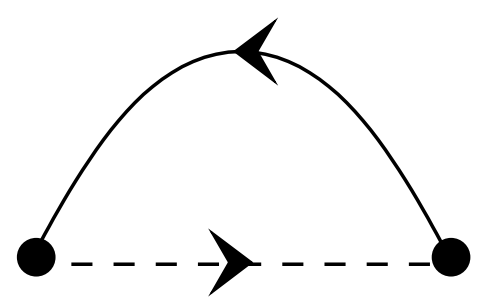

FIG. 2. NCA self-energy diagrams for (a) pseudo fermion and (b) slave boson. The solid line represents the propagator $\tilde{D}$, the Green's function of the active $\operatorname{dot} A$ when $W=0$. The dashed (wavy) line is the pseudo fermion (boson) propagator. The solid circle means the tunneling matrix $W$. 

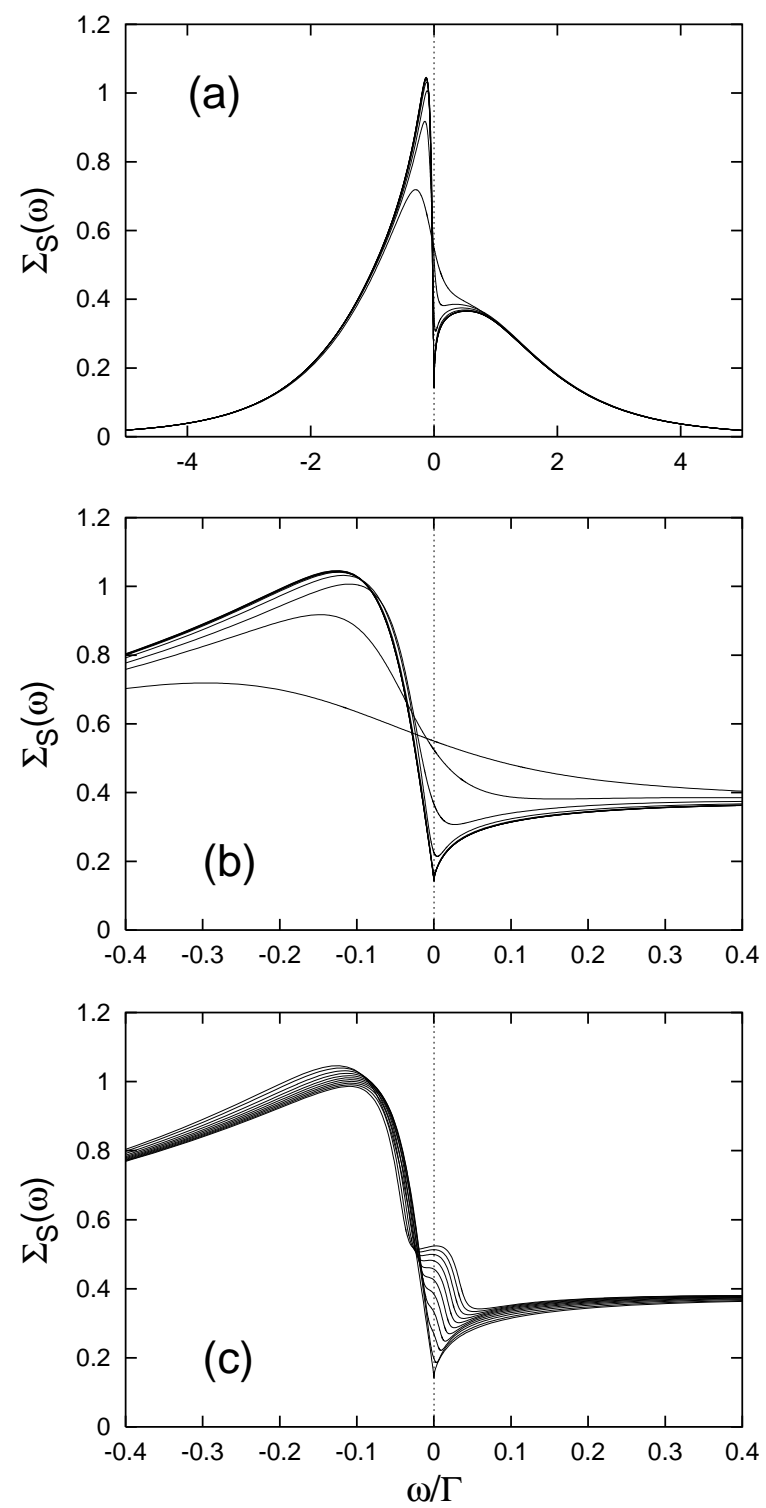

FIG. 3. Temperature and bias voltage dependence of the self-energy of a side-connected dot $S$. Temperature variation of the self-energy is displayed in (a). The panel (b) is the magnified view of the panel (a) near $\omega=0$. With decreasing temperatures the structure in the self-energy becomes sharper near $\omega=0$. Temperature variations are $T / T_{K}=31.6,10,3.16,1,3.16 \times 10^{-1}, 10^{-1}, 3.16 \times 10^{-2}, 10^{-2}, 3.16 \times 10^{-3}, 10^{-3}$. At $T=10^{-3} \times T_{K}$ the variation of the self-energy with increasing bias voltage is displayed in (c). The source-drain bias voltages are varied from $\mathrm{eV}=0$ to $\mathrm{eV}=20 \times T_{K}$ by the amount $2 \times T_{K}$. 

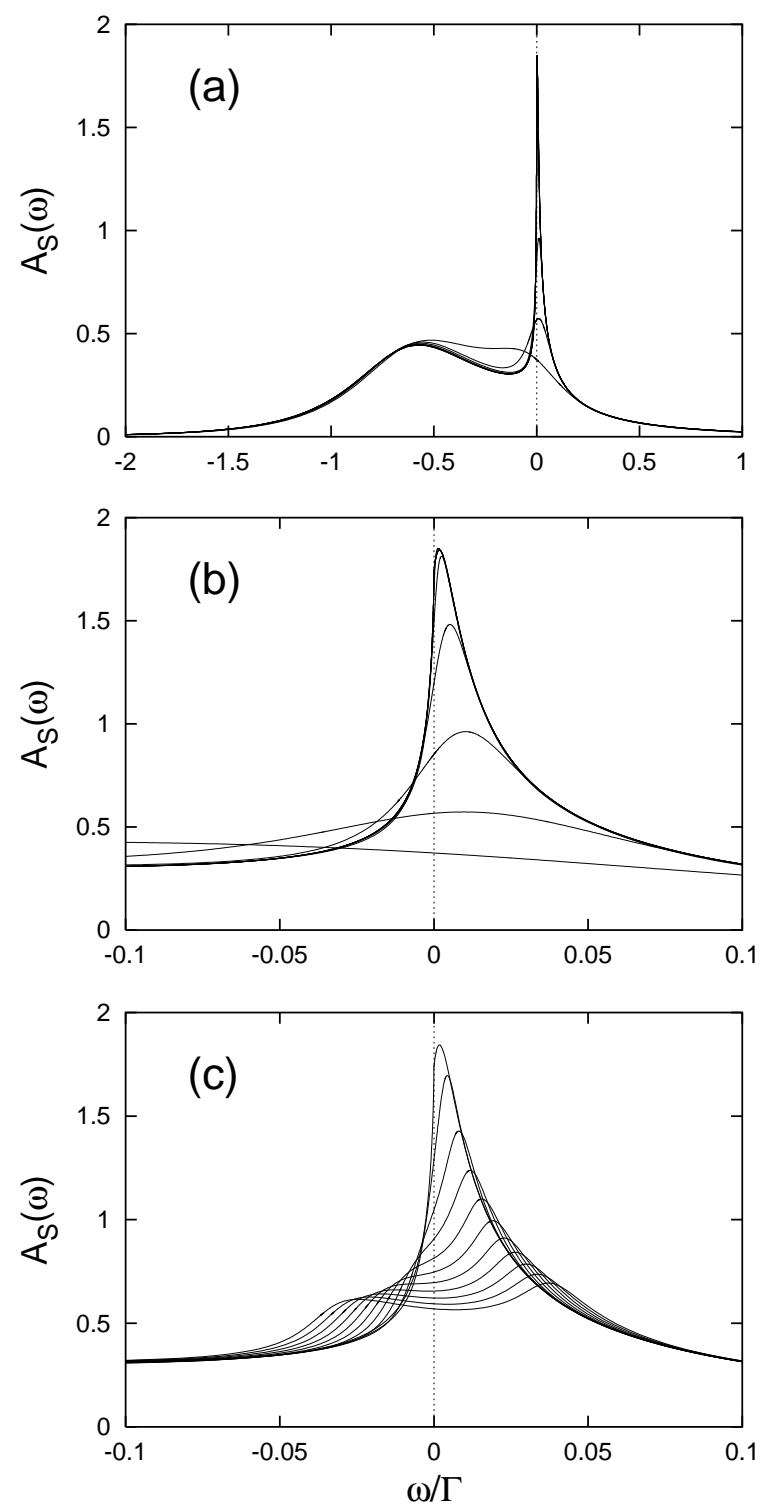

FIG. 4. Temperature and bias voltage dependence of the spectral function $A_{S}(\omega)$ at a side-connected dot $S$. (a) and (b) show the developments of a Kondo resonance peak with decreasing temperature. The panel (b) is a magnified view of the panel (a). The bias voltage dependence of the spectral function $A_{S}(\omega)$ at $T=10^{-3} \times T_{K}$ is displayed in (c). The Kondo resonance peak is progressively suppressed with the increasing bias voltage and develops into two-peak structure. Temperature and voltage variations are the same as in Fig. 3. 

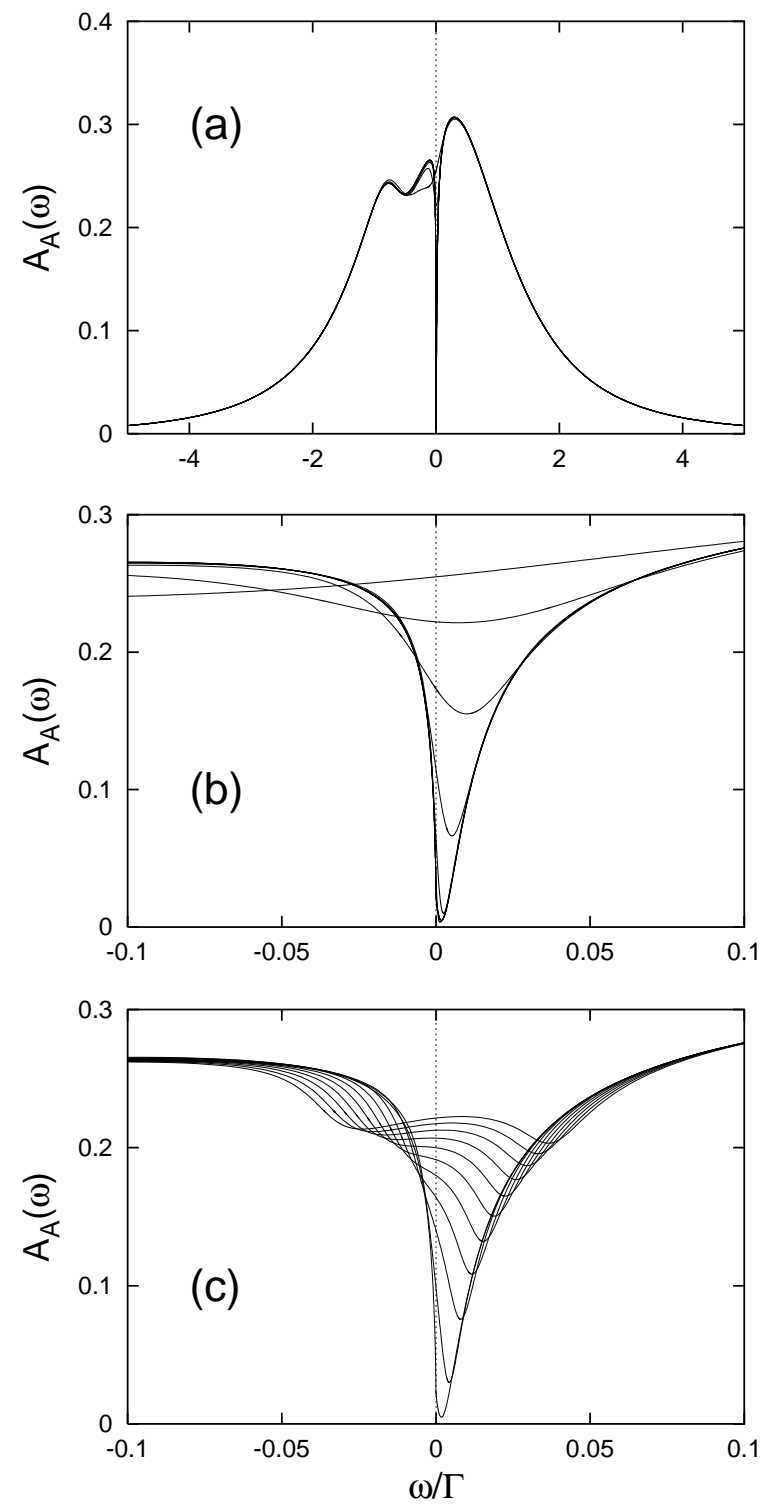

FIG. 5. Temperature and bias voltage dependence of the spectral function $A_{A}(\omega)$ at the active dot $A$. Due to the Fano interference the spectral function $A_{A}(\omega)$ becomes depleted near $\omega=0$ as the temperature is lowered below $T_{K}$, as shown in (a) and (b). When a finite bias voltage is applied, the spectral depletion is refilled and the two-peak structure in $A_{S}(\omega)$ is manifested as the double-dip structure in $A_{A}(\omega)$. Temperature and voltage variations are the same as in Fig. 3 . 


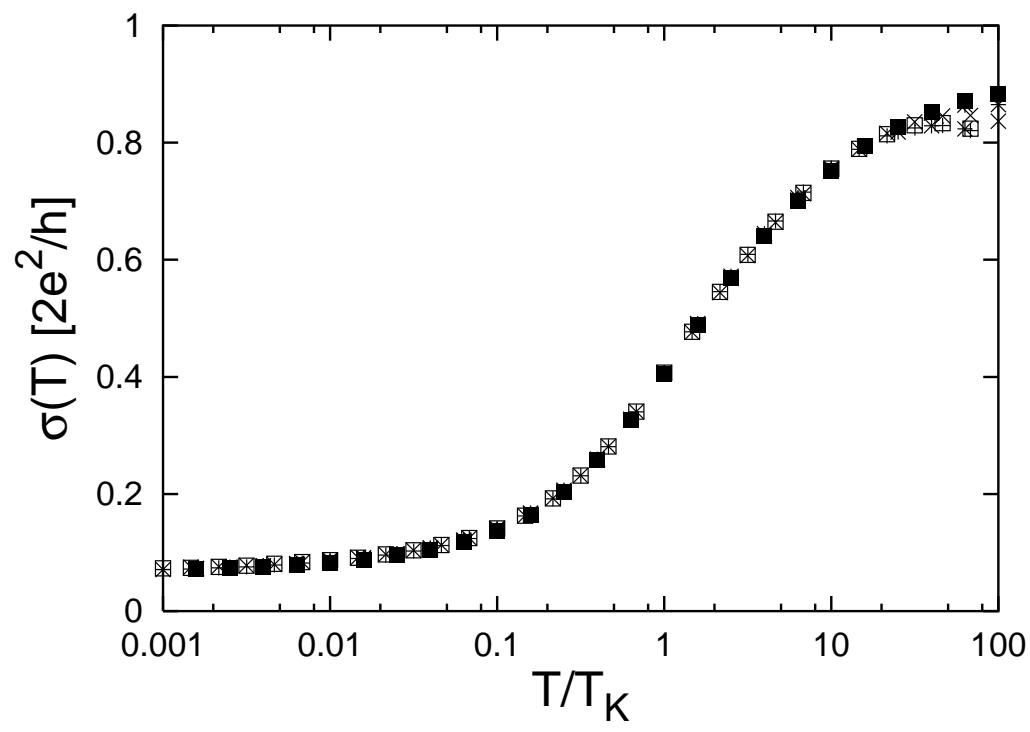

FIG. 6. Temperature dependence of linear response conductance $\sigma(T)$. The conductance at $V=0$ is suppressed with decreasing temperature as can be expected from the temperature dependence of the spectral function $A_{A}(\omega)$. The conductance is suppressed logarithmically due to the enhanced Kondo scattering near the Kondo temperature and becomes saturated with decreasing temperature. $\sigma(T)$ shows the scaling behavior over a wide range of temperature. Symbols mean different sets of model parameters or differing Kondo temperatures.

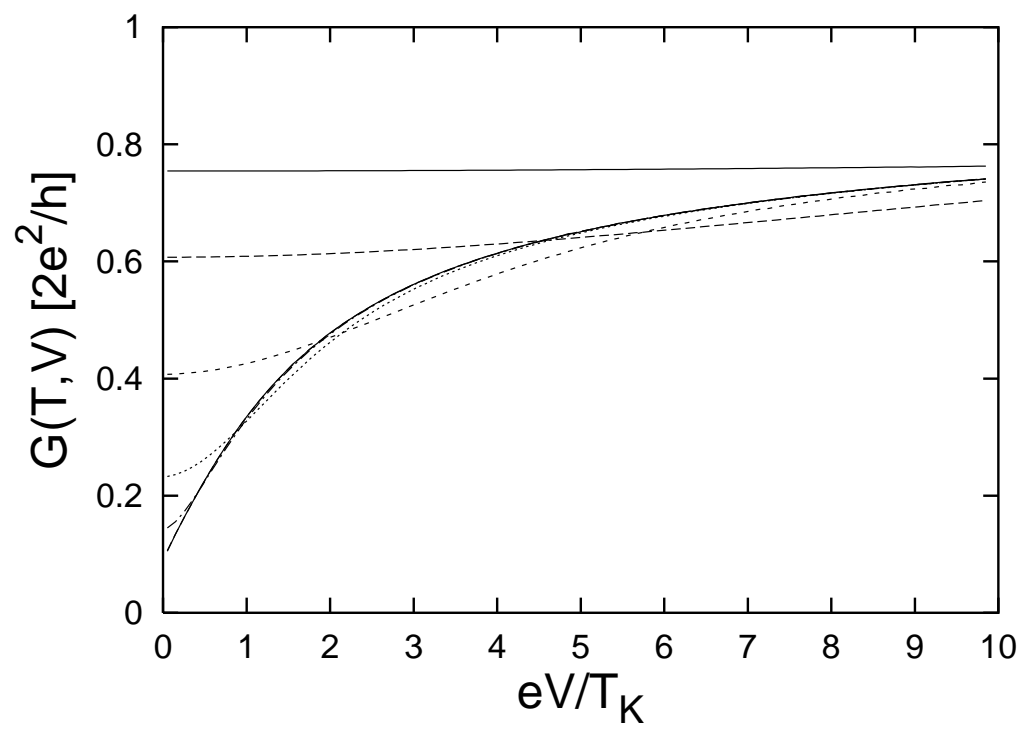

FIG. 7. Differential conductance $G(T, V)=d I / d V$ with varying temperature. The differential conductance is progressively suppressed near zero bias voltage with lowering temperature. From top, the temperature variations are $T / T_{K}=10,3.16,1,3.16 \times 10^{-1}, 10^{-1}, 3.16 \times 10^{-2}, 10^{-2}, 10^{-3}$. The last three curves cannot be distinguished with the naked eye. 

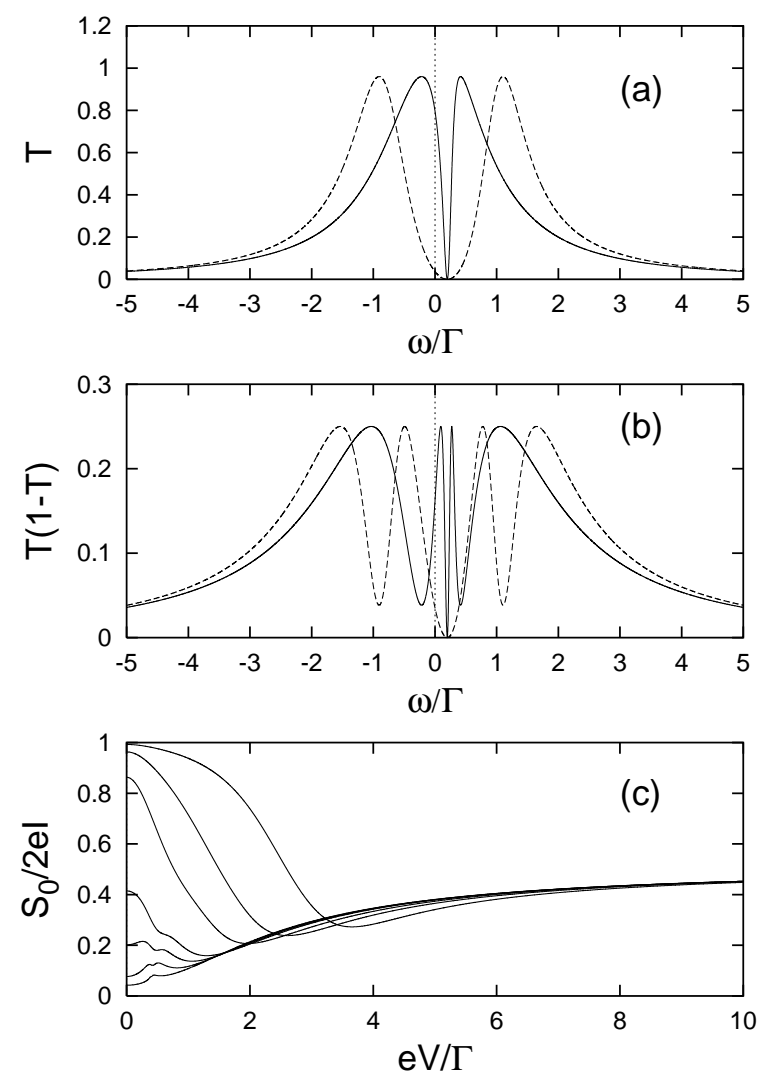

FIG. 8. Tunneling coefficients and Fano factor at $T=0 \mathrm{~K}$. Due to the Fano interference the transmission coefficient $T(\omega)$ is completely suppressed when $\omega=E_{S}$, the energy level of the side-connected dot. The Fano factor, $F=S_{0} / 2 e I$, has a dip structure accordingly. The model parameters are $E_{A}=0, E_{S} / \Gamma=0.2$ for displayed curves. In (a) and (b), $W / \Gamma=0.3(1.0)$ for solid(dashed) line, respectively. In panel (c), the values of $W / \Gamma$ are varied from the bottom successively as $0.1,0.2,0.3,0.4,0.7,1.0,1.5$. 